\title{
El proceso de aprendizaje virtual y su incidencia en la salud mental de los estudiantes
}

\author{
Fecha de recepción: 2021-09-15 • Fecha de aceptación: 2021-11-08 • Fecha de publicación: 2022-01-10
}

\author{
Lizbeth Aracely Toasa Guachi' \\ Universidad Tecnológica Indoamérica, Ecuador \\ liztoasa@gmail.com \\ https://orcid.org/0000-0002-6458-6608
}

Renato Mauricio Toasa Guachi²

Universidad Tecnológica Israel, Ecuador

rtoasa@uisrael.edu.ec

https://orcid.org/0000-0002-2138-300X

\section{Resumen}

El proceso de aprendizaje y enseñanza evoluciona con el pasar del tiempo, debido a esto se implementan distintas metodologías y técnicas para que los estudiantes aprendan de manera óptima; sin embargo, una de las problemáticas más comunes dentro de la salud mental es el estrés y la ansiedad, que pueden generarse al no adaptarse a los nuevos sistemas en la educación, como lo es el caso del aprendizaje virtual obligado por la pandemia por COVID-19. En este contexto, la presente investigación busca describir la percepción de los estudiantes de octavo semestre de la carrera de psicología de la Universidad Tecnológica Indoamérica de la ciudad de Ambato, respecto a su experiencia de aprendizaje en entornos virtuales. Para ello, se recogen las percepciones de una muestra de 50 estudiantes de la Facultad de Ciencias Humanas y de la Salud, basándose en su experiencia con las clases bajo la modalidad virtual en tiempos de pandemia. Los resultados muestran que los estudiantes consideran que el estrés influye en su aprendizaje, y que suelen generar rasgos ansiosos al realizar tareas complejas. La solución hacia los problemas se direcciona al poder conocer los niveles de estrés y ansiedad que pueden tener los estudiantes, el docente influye mucho, por lo que él podrá utilizar metodologías interactivas e incorporar pausas activas, 
disminuyendo los niveles de estas patologías, con el fin de estimular las habilidades y capacidades de los alumnos.

\title{
Palabras clave: aprendizaje virtual, estudiantes, salud mental, estrés, ansiedad
}

\begin{abstract}
The learning and teaching process evolves over time, due to this different methodologies and techniques are implemented for students to learn optimally; however, one of the most common problems within mental health is stress and anxiety, which can be generated by not adapting to new systems in education, as is the case of virtual learning forced by the pandemic by COVID-19. In this context, this research seeks to describe the perception of eighth semester students of psychology at the Universidad Tecnológica Indoamérica in the city of Ambato, regarding their learning experience in virtual environments. For this purpose, the perceptions of a sample of 50 students of the Faculty of Human and Health Sciences are collected, based on their experience with classes under the virtual modality in times of pandemic. The results show that students consider that stress influences their learning, and that they tend to generate anxious traits when performing complex tasks. The solution to the problems is directed to know the levels of stress and anxiety that students may have, the teacher has a great influence, so he can use interactive methodologies and incorporate active breaks, reducing the levels of these pathologies, in order to stimulate the skills and abilities of students.
\end{abstract}




\section{Introducción}

En la actualidad, debido a los grandes cambios en los procesos educativos, es de suma importancia reconocer que el proceso de aprendizaje permite que los estudiantes adquieran conocimientos y habilidades dentro y fuera del entorno educativo, obteniendo una adecuada formación profesional basándose en sus experiencias. Para generar un óptimo proceso de aprendizaje, ya sea en escuelas, colegios o universidades, se tomará en cuenta la relación e interacción entre el profesor y el alumno, creando un ambiente de participación y teniendo en cuenta elementos como la motivación y los sentimientos, con el fin de resolver dudas y problemas, es así que todo tipo de aprendizaje da paso a la asimilación de nuevos conceptos que se pondrán en práctica, de manera que se logrará retener la información a largo plazo (Escobar, 2015).

Al ser un proceso complejo, es necesario seguir fases que se complementan unas con otras, por esta razón, el proceso idóneo para un correcto aprendizaje debe tener nueve fases esenciales según lo menciona Yánez (2016) en su investigación sobre "El proceso de aprendizaje: fases y elementos fundamentales".

La motivación es la primera, esta juega un papel fundamental en los estudiantes por el deseo de aprender de manera rápida y eficiente, sienten un impulso por las necesidades personales y las perspectivas del futuro, aquí el docente brindará estrategias pedagógicas e impulsará a sus alumnos positivamente; posteriormente, el mantener el interés por aprender, mostrando niveles altos de concentración y atención con el fin de alcanzar sus metas y objetivos planteados; por consiguiente, la atención es la fase siguiente, esta permite la interpretación de lo que va aprendiendo con precisión y claridad; se continúa con la cuarta, fase siendo la adquisición de conocimientos, una herramienta que ayuda en la resolución de problemas al desarrollar la inteligencia con base en la construcción de ideas relacionadas con otras (Yánez, 2016).

Al involucrar el pensamiento para propiciar la memoria significativa, esta va de la mano con la comprensión de conceptos y la interiorización, implicando la organización y el entendimiento de la información; esto permite que se produzca la asimilación, aquí se necesita almacenar los aspectos positivos de las experiencias que den paso a que los conocimientos adquiridos sean por largo plazo; facilitando la aplicación de todo lo que se ha aprendido durante el proceso: es decir, pondrán en práctica los conceptos, volviéndose dinámicos y fáciles de comprender; con el fin de conseguir una transferencia, lo que significa resaltar el aprendizaje demostrativo al construir nuevas enseñanzas con la ayuda de conocimientos previos, ayudando en la instrucción de otras personas con lo aprendido a lo largo del tiempo; finalmente la última etapa de este proceso es la evaluación, se resalta la observación y la interpretación de los resultados de parte del docente, haciendo que los alumnos adquieran nuevas aptitudes, destrezas y valoren dentro de su formación académica (Yánez, 2016).

Es necesario comprender que la neurociencia impulsa el proceso de aprendizaje de las personas, hacia una adecuada adquisición de conocimiento a través de la experiencia, la observación y la experimentación. Esta ciencia está revolucionando el ámbito de la educación al poder conocer los procesos biológicos que facilitan el aprendizaje y el descubrir cómo se almacena la información 
en el cerebro, permitiendo implementar métodos de enseñanza que faciliten la adquisición del conocimiento (Terigi, 2016). Al saber cómo intervienen los procesos neurobiológicos en el aprendizaje, permitirá que este sea óptimo y eficaz.

\subsection{Importancia de la psicología del aprendizaje}

El aprendizaje es un proceso mediante el cual una persona adquiere conocimientos, actitudes y habilidades a través de la experiencia y la enseñanza, existen diferentes teorías para explicar la forma de aprender, una de ellas es la psicología conductista, esta describe que se adquiere mediante la observación de la conducta de un individuo.

Otra postura es la de aprender por imitación; es decir, que si se repite un proceso observado podemos adquirir conocimientos, implica el tiempo y habilidades, mismas que permiten retener información y serán usadas en el desarrollo personal y social (Pellón et al., 2015). Estudia el proceso de aprendizaje del ser humano donde se visualiza cambios de carácter transitorio o permanente, enfocándose en el proceso continuo en el que un sujeto adquiere nuevos conocimientos, comparando los comportamientos simples y naturales de un niño como los comportamientos de un adulto, además, de que se puede observar como los sentimientos, habilidades y pensamientos son diferentes y precisamente el aprendizaje es el elemento que marca la diferencia en el proceso aprendizaje-enseñanza (Ardila, 2001).

\subsection{Teorías del aprendizaje}

En este apartado se mencionan las 4 teorías que se presentan en la investigación.

A. La teoría conductista en el aprendizaje se centra en la adquisición comportamientos nuevos a través de un estímulo que genera una respuesta positiva o negativa, por esta razón, los conductistas estudian el aprendizaje centrándose en conductas abiertas que pueden ser observadas y medidas por externos del aprendizaje, o por estímulos que producen respuestas porque el ser humano está determinado por su entorno y la única manera de entender su comportamiento es a través de estímulos de las conductas observables. En lo que piensa el conductismo es en conseguir una conducta determinada fundamentada en el estudio de experiencias internas o sentimientos por medio de métodos mecanizados, donde el docente funciona como alfarero que moldea la conducta del estudiante (Cepeda et al., 2018).

Cuenta con dos variables importantes, la primera es el condicionamiento clásico descrito por Iván Pávlov, utiliza un modelo estímulo-respuesta, siendo un tipo de aprendizaje en la educación, donde el estímulo neutro desencadena una respuesta al asociarse con un estímulo incondicionado, de manera que ayudará al docente en el proceso de enseñanza y aprendizaje para moldear un aprendizaje efectivo en los estudiantes (Bertrán, 2018), la segunda variable es el condicionamiento operante, este intenta modificar la conducta por medio de estímulos para conseguir una conducta deseada, suprimiendo comportamiento negativos, es decir, en el ámbito educativo los docentes tratan de modificar conductas negativas de los estudiantes, motivándolos al no darles castigos si realizan sus tareas o al darles recompensas por su buen comportamiento (Granda, 2016). 
B. La teoría cognitivista se encarga del estudio de la condición y función de los procesos mentales implicados en el conocimiento de un individuo, su objetivo es estudiar los mecanismos básicos y profundos con los que se elabora el conocimiento para poder almacenar, recuperar y recibir información a través de los sentidos. El aprendizaje según el cognitivismo constituye la síntesis de la forma y el contenido recibido en las percepciones, las cuales actúan de forma relativa y personal, se encuentra fluida por los antecedentes del aprendizaje en una visión común, vista mucho más simple que el cambio observable en el comportamiento humano (Cáceres \& Munévar, 2017).

C. La teoría humanista propone un aprendizaje significativo y vivencial, lo define como el proceso que modifica la percepción de los individuos que derivan de la reorganización del y; en este sentido, este tipo de aprendizaje es puramente mental y afectivo, solo se enfoca en el estudiante y en que se obtenga el conocimiento necesario, contando con una potencialidad natural para el aprendizaje (López, 2018). El estudiante percibe el tema de estudio como importante en sus propios objetivos, la mayor parte se logra mediante la práctica, esto significa que el estudiante debe enfrentar problemas prácticos, por eso el humanismo plantea que el docente sea un facilitador y un guía del aprendizaje, presentando actitudes como la de ser auténtico frente a sus alumnos y apreciarlos, proponiendo un aprendizaje significativo y vivencial mediante un proceso que modifica la percepción de los estudiantes, haciendo que tengan libertad para alcanzar y confiar en sí mismos (Varona, 2016).

D. La teoría del aprendizaje social se apoya en la idea de que los estudiantes aprenden en entornos sociales por medio de la observación y la imitación de un comportamiento, aquí el sujeto se ve afectado por la influencia de otros, se menciona que sería muy peligroso si las personas aprendieran basándose en su experiencia como se creía anteriormente. También se afirma que los procesos cognitivos se dan al interiorizar un nuevo conocimiento, dichos conocimientos se deben gracias al contexto en el que se produce, es por esto que la teoría es considerada como uno de los primeros puentes entre la corriente conductista y la cognitiva, en el campo de la psicología, ya que se deben incorporar elementos como refuerzo, la motivación y la atención (Vega et al., 2019).

\subsection{Alteraciones en la salud mental de los estudiantes}

Según la Organización Mundial de la Salud (2001), la salud mental es "un estado de bienestar en el cual el individuo se da cuenta de sus propias aptitudes, puede afrontar las presiones normales de la vida, puede trabajar productiva y fructíferamente y es capaz de hacer una contribución a su comunidad", es decir, es el estado de bienestar de las personas para hacer frente al estrés normal de su diario vivir. En la actualidad, la pandemia por Covid-19 repercutió en la vida de todas las personas, cambiándola de manera repentina, se implementaron nuevas modalidades de estudio como las clases virtuales, la enseñanza en casa es difícil para los estudiantes, ya que algunos de ellos, al no tener un tutor en la presencialidad, no tienen un buen aprendizaje generándoles estrés y rasgos ansiosos.

Los estudiantes universitarios han sido la población más vulnerable al sentir estrés y ansiedad por las clases virtuales, su salud mental se ha visto afectada al no poder aprender de manera 
adecuada, dejando de lado el optimismo que mostraban en la presencialidad. Se debe tomar en cuenta los factores estresantes como la presión de los exámenes, entrega de tareas, falta de apoyo de la familia y el sentir presión por los docentes que exigen demasiado, pero no tienen buenas metodologías de enseñanza, todos estos factores desencadenan estrés y ansiedad en el ámbito académico viéndose afectada su salud mental por la alteración de sus emociones y sentimientos al verse perdidos por no comprender las clases y por consecuente podrían tener dificultad al aprobar los semestres (Cano et al., 2020).

Existen trabajos relacionados que sustentan lo mencionado anteriormente:

Lovón \& Cisneros (2020) afirman en su trabajo que "la salud mental del alumnado en la universidad se ve aún más alterada por una forzosa virtualización de la enseñanz,a no esperada y menos aún planificada".

Mientras que Aguilar et al. (2020) mencionan que "la educación a distancia, refiriéndose a modalidades de aprendizaje que hacen uso de plataformas virtuales de aprendizaje, así como de videoconferencias (...) el uso y aplicación de la tecnología, ha transformado el proceso de la comunicación profesor-estudiante y estudiante-estudiante en un ir y venir interactivo. Sin embargo, esto ha sumado un cierto grado de estrés a lo habitual, el estrés es una reacción adaptativa del organismo ante las demandas de su medio, cuando estas demandas se originan dentro de un medio educativo".

Por su parte, los autores Afkri \& Hosseni (2021) sostienen que "debido a esta pandemia y sus consecuencias, los estudiantes experimentaron momentos únicos de cuarentena domiciliaria y educación virtual. Por lo tanto, comprender las complejidades, las experiencias, las creencias y las actitudes hacia la práctica de la cuarentena domiciliaria y el cambio nuevo y repentino a la educación virtual, en lugar de las clases presenciales, es crucial y conduciría a que los formuladores de políticas educativas y el personal de salud comprendan de manera integral las consecuencias psicológicas de la educación virtual y cuarentena".

Por otro lado, existen fases que permiten identificar el nivel de estrés, en este sentido, Hans Selye en 1936, las describe de esta manera:

La fase de alarma hace que el organismo empiece a desarrollar alteraciones psicológicas y fisiológicas, suelen presentar cierta sintomatología como sudoración, dolores de cabeza, tensión muscular, taquicardia y aumento de la tensión arterial y la frecuencia respiratoria. Al ser una fase de poca duración, no es perjudicial, ya que el organismo dispondrá un tiempo para recuperarse de los estresores; cuando el organismo no cuenta con el tiempo para recuperarse inicia la fase de resistencia, al hacer frente a la situación de la mejora manera, presentando una disminución del rendimiento, desorganización considerable en su diario vivir y suelen mostrar poca tolerancia en momentos frustrantes; finalmente, la fase de agotamiento cuenta con la energía de adaptación limitada, es decir, si el estrés continúa con más intensidad el organismo podría entrar en la fase de agotamiento y presentar alteraciones fisiológicas, psicológicas psicosociales suelen ser crónicos y en casos extremos hasta irreversibles (Tan \& Yip, 2018). 
Para poder identificar los tipos de ansiedad hay que tomar en cuenta su composición estructural, se parte de los procesos relevantes alrededor de 3 niveles: el nivel más bajo, relacionado con mecanismos cognitivos, asociándose con la memoria atencional y de trabajo, y los mecanismos somáticos, centrándose en sistemas afectivos y motivacionales; el nivel medio se ve perjudicado en la disminución del campo de la percepción y una alteración en sus relaciones sociales, generando así preocupaciones y tensiones considerables en su diario vivir; finalmente el nivel más alto indica marcadores de comportamiento, presentando ataques de pánico y estados altos de estrés, repercutiendo en el ámbito educativo y personal por un miedo intenso (Fajkowska et al., 2017).

El estrés se asocia con la pandemia por Covid-19, causando una disminución de la motivación y por ende del mismo rendimiento académico, generando niveles considerables de síntomas ansiosos por la tensión emocional y física lo que provoca frustración de los estudiantes al no poder adaptarse a las metodologías y al ritmo de estudio de los demás compañeros (González, 2020).

En la siguiente Tabla 1 se planteó la siguiente escala para medir el nivel de estrés y ansiedad de los estudiantes universitarios, al identificar 3 fases de estrés y 3 niveles de ansiedad.

Tabla 1.

Niveles de estrés y ansiedad en estudiantes

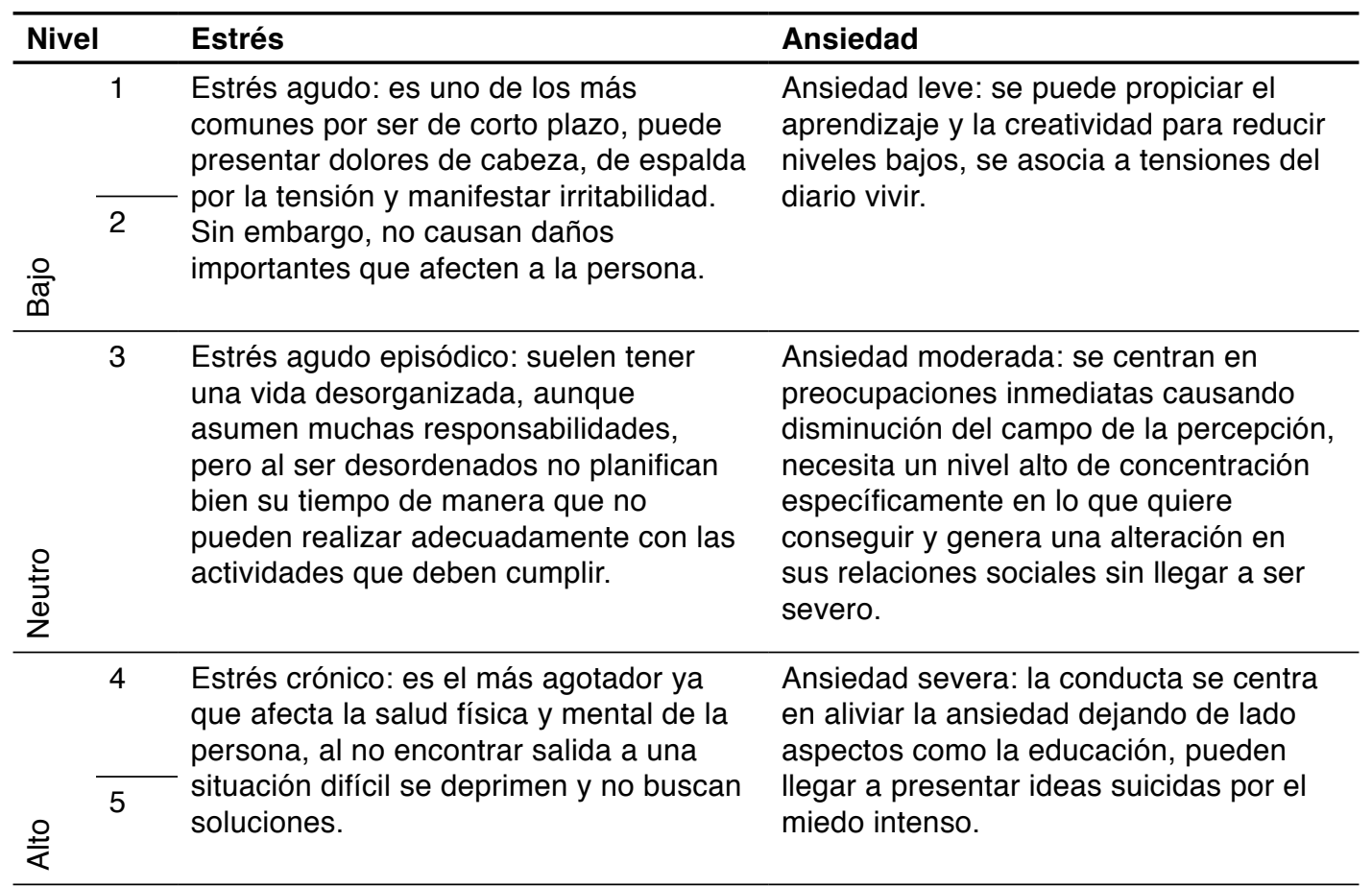

\subsection{Aportes de la neurociencia hacia el aprendizaje}

Los procesos de enseñanza y aprendizaje a lo largo del tiempo han ido evolucionando, conforme a los nuevos avances que aporta la neurociencia, algo que resalta en estos procesos es el rol del docente, que no se limita en impartir conocimientos, sino también el generar una interacción 
con los alumnos, para poder generar un buen aprendizaje es necesario implementar estrategias innovadoras, de manera que se despierte el interés y la motivación de los alumnos, permitiendo así un óptimo desarrollo integral en la educación, al no generar factores estresantes (Araya \& Espinoza, 2020). Con lo expuesto anteriormente nace una nueva visión sobre los aportes de la neurociencia, como lo es la neuroeducación y la neurolingüística.

\section{A. Neuroeducación}

Según Béjar (2014), es un nuevo enfoque de enseñanza basada en el cerebro humano, permite conocer cómo funciona en integración de la psicología, la educación y la neurociencia, con el fin de potenciar los procesos de aprendizaje y memoria al implementar nuevas estrategias y técnicas educativas. Con la neuroeducación (Figura 1), al diseñar estas estrategias de manera eficiente y efectiva en la enseñanza, se logrará que el docente brinde sus conocimientos con nuevas metodologías que permitan potenciar el desarrollo de las habilidades de los alumnos (Gómez \& Vázquez, 2018).

Figura 1.

Componentes de la Neuroeducación

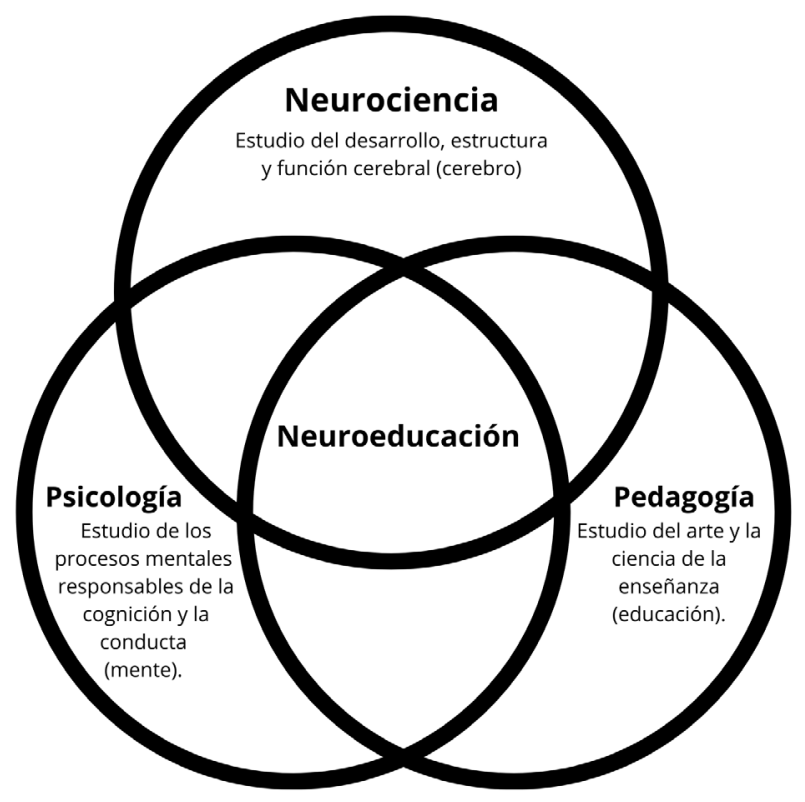

Fuente: (Ramos, s.f.)

\section{B. Neurolingüística}

Es una de las áreas de la neurociencia que va de la mano con la psicología y la lingüística, por su nivel de complejidad en el desarrollo del cerebro, siendo un conjunto para determinar los mecanismos del lenguaje y qué trastornos del aprendizaje podrían detener su buen 
funcionamiento. Por esto es necesario una adecuada programación de la neurolingüística, se parte de la psicología, ya que se encarga de analizar y modificar conductas a través del estudio del lenguaje verbal, corporal y gestual, además, se apoya de la lingüística, al ser una disciplina que estudia netamente las estructuras esenciales en el lenguaje, con el fin de conseguir que los conocimientos adquiridos en las personas sean significativos al tener un adecuado aprendizaje (Rojas, 2017).

En base a todo lo expuesto anteriormente, este trabajo propone un estudio que pretende proporcionar un autorreporte de los niveles de estrés y ansiedad en entornos virtuales en periodo de pandemia por COVID-19, según la percepción de los estudiantes universitarios, ya que fueron los principales afectados al tardarse mucho tiempo en adaptarse a esta nueva modalidad de enseñanza.

El resto del documento se encuentra dividido en las siguientes secciones, sección 2 muestra la metodología y la literatura encontrada en este estudio, sección 3 muestra los resultados y finalmente las conclusiones se presentan en la sección 4.

\subsection{Trabajos relacionados}

El proceso de aprendizaje y la salud mental de los estudiantes siempre ha estado estrechamente relacionados, esto lo demuestra la literatura encontrada en distintas bases de datos de alto impacto, y se mencionan a continuación. Inicialmente, Blanco et al. (2017) desarrollan un estudio enfocado en la neurociencia del aprendizaje asociativo de mamíferos, donde mencionan que: "el aprendizaje asociativo de los mamíferos se organiza en sistemas funcionales separados definidos anatómicamente", presentan además una descripción la orientación que ha tomado la neurociencia del aprendizaje y se revisan algunos de los avances realizados dentro de ese importante campo de estudio.

Por otro lado, Cabeza et al. (2018) proponen un estudio orientado en el análisis de la neurociencia cognitiva y la memoria, mencionan que la neurociencia como tema de estudio crece considerablemente rápido, a lo que sustentan con que "este crecimiento ha sido estimulado por dos desarrollos importantes. En primer lugar, la biología molecular ha transformado la neurobiología celular y ha dado lugar a un nuevo marco conceptual para la señalización, un marco molecular que abarca no solo la señalización en las células nerviosas sino en todas las células del cuerpo. En segundo lugar, el trabajo sobre el cerebro y la cognición, que tradicionalmente se asociaba con varias disciplinas diferentes, se ha fusionado en una sola disciplina: la neurociencia cognitiva. Esto ha proporcionado un nuevo marco para el estudio de la memoria, la percepción, la acción, el lenguaje y quizás incluso la conciencia”.

Bishop \& Gagne (2018) realizan un trabajo sobre el aprendizaje y la memoria desde la perspectiva de la computación neuronal, y sostienen que varios científicos a nivel mundial "a menudo dividen el aprendizaje en tres clases amplias: aprendizaje supervisado, reforzado y no supervisado. Las tres clases comparten el objetivo común de formar representaciones almacenadas para luego recordarlas y utilizarlas para guiar el comportamiento; difieren en los mecanismos específicos por los cuales las representaciones almacenadas se derivan de la experiencia". Basan su estudio en 
analizar estos tres tipos de aprendizaje a nivel conductual utilizando un ejemplo concreto: la tarea que enfrenta un niño pequeño al aprender el nombre de un objeto experimentado visualmente en el entorno.

La pandemia COVID-19 afectó mucho al sector educativo y al proceso de aprendizaje, Ramirez (2020) menciona en su investigación que "la pandemia de Covid-19 desafió a los profesores a innovar con cursos de aprendizaje remoto", este trabajo revisa los modelos curriculares y las estrategias pedagógicas destinadas a mejorar las experiencias educativas afrontando los nuevos retos del siglo $\mathrm{XXI}$.

Por su parte, Sandrone \& Schneider (2020) mencionan que "el brote pandémico de SARS-CoV2 está acelerando las transformaciones educativas en curso y se están cuestionando los modelos educativos tradicionales. Con el distanciamiento social y la incertidumbre sobre el momento de la reapertura del campus físico, existe una necesidad imperiosa de una experiencia educativa más flexible. Esto afecta la educación en neurociencias en todos los niveles, desde la licenciatura hasta los estudios de posgrado y la educación continua."

La revisión de literatura detallada en párrafos anteriores evidencia que el trabajo propuesto puede generar un impacto importante en la comunidad científica y académica, ya que los trabajos encontrados se orientan en la neurociencia y su relación con la educación y el aprendizaje; sin embargo, no se ha evidenciado algún estudio que se oriente al impacto en los estudiantes universitarios, como lo que propone este trabajo de investigación.

\section{Metodología}

Fue esencial dentro de la investigación la revisión bibliográfica, al ser una metodología que permite recopilar información relevante sobre temas específicos, con el fin de analizarla de manera profunda, al ser la etapa primordial de proyectos de investigación, permite realizar un análisis detallado con la ayuda de repositorios, en donde se almacenan artículos científicos y libros (Martín \& Lafuente, 2017). Se la realiza a través de consultas bibliográficas citadas, siendo la información sumamente clara y alcanzando con éxito las necesidades de los investigadores, además, en el proceso de investigación se toma en cuenta la información basada en libros y revistas científicas, es así que la búsqueda bibliográfica brindará información verídica que aporte al estudio (Gómez et al., 2016).

Igualmente se utilizó la metodología cuantitativa para la recolección de datos, basándose en la medición numérica con el fin establecer pautas de comportamiento y probar hipótesis, este enfoque deriva objetivos y formula preguntas de investigación que dan paso a establecer hipótesis y determinar variables. Al poder analizar las mediciones obtenidas se pueden obtener una serie de conclusiones respecto de la o las hipótesis planteadas (Hernández et al., 2014). En este trabajo se utilizó como instrumento una encuesta de 6 preguntas acerca del estrés y los rasgos ansiosos que puede provocar el aprendizaje virtual en tiempos de pandemia por Covid-19, esta fue aplicada a estudiantes universitarios, ya que fue una de las poblaciones más afectadas, repercutiendo en su aprendizaje. 
La muestra a la que está direccionada este trabajo es una parte de la comunidad de la Universidad Tecnológica Indoamérica de la ciudad de Ambato, constituida por 50 estudiantes, siendo el total de octavo semestre de la carrera de psicología, contando con edades de entre 21 y 25 años. Hay que considerar que, al ser de los niveles superiores de la carrera, los estudiantes dominan perfectamente los conceptos de estrés y ansiedad.

\section{Resultados}

La técnica utilizada se realizó de manera virtual en el formulario de Google, para obtener datos de manera rápida y eficaz. Esto permitió identificar el nivel de estrés y rasgos ansiosos que les genera el implementar nuevas metodologías de aprendizaje virtual, utilizando plataformas como Zoom, Teams, el aula virtual Canvas y el sistema gestión académico, el mismo que permite el acceso a la biblioteca virtual.

Constituye un objetivo del presente estudio describir la percepción de los estudiantes de octavo semestre de la carrera de psicología de la Universidad Tecnológica Indoamérica de la ciudad de Ambato, respecto de su experiencia de aprendizaje en entornos virtuales.

En la siguiente Figura 2 se evidencian los resultados de la Pregunta 1: del 1 al 5 indique el nivel de ansiedad que le generan las clases virtuales.

Figura 2.

Nivel de ansiedad que generan las clases virtuales

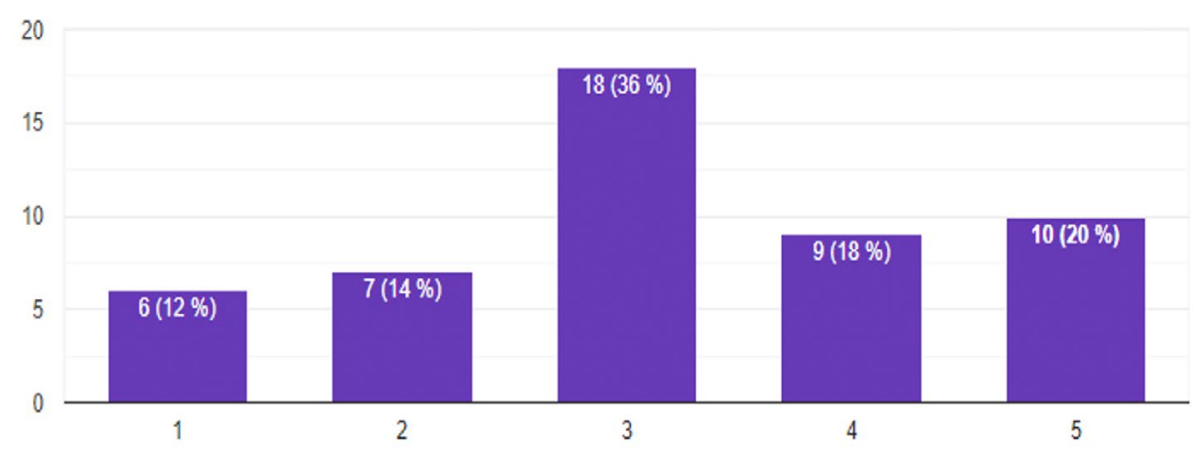

De los 50 estudiantes universitarios a los que se les aplicó la encuesta sobre estrés y ansiedad ante nuevas metodologías que aportan la neurociencia al aprendizaje, se puede evidenciar que el $36 \%$ respondió que su nivel de ansiedad sería 3 siendo neutral; un $20 \%$ con un nivel de 5 , el $18 \%$ corresponde a un nivel 4 , demostrando niveles altos; por otro lado, el $14 \%$ representa un nivel 2 y finalmente el 12\% cuenta con un nivel 1, indicando niveles bajos, todo esto en relación con la ansiedad que les genera las clases virtuales. Actualmente la pandemia ha obligado a los estudiantes a recibir clases de manera virtual, generando rasgos ansiosos por algunas causas como el no saber utilizar de manera adecuada las herramientas tecnológicas, el no tener una buena conectividad de Internet o en varios casos el no contar con aparatos tecnológicos en su 
hogar, teniendo que acudir a bibliotecas o centros de cómputo con varias personas creando un miedo por posibles contagios.

¿En qué nivel considera que el estrés influye en su aprendizaje?, fue la segunda pregunta que se realizó, en la siguiente Figura 3 se representan los porcentajes.

\section{Figura 3.}

Nivel de estrés en el aprendizaje

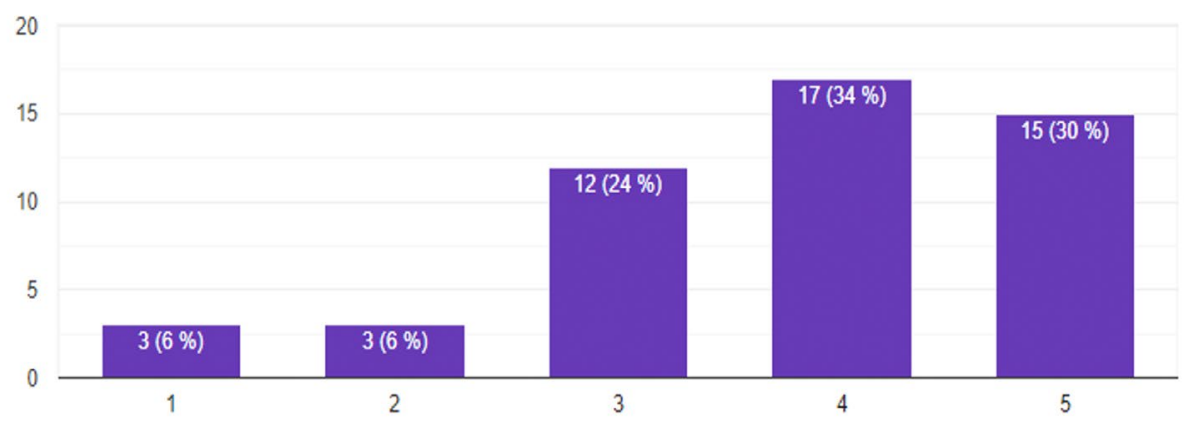

De acuerdo al gráfico anterior se puede evidenciar que el 34\% respondió que su nivel de estrés sería 4 , un $30 \%$ con un nivel de 5 , siendo niveles altos; el $24 \%$ corresponde a un nivel 3 , demostrando un nivel neutro; por otro lado, el $6 \%$ representa un nivel 2 y finalmente el $6 \%$ cuenta con un nivel 1, indicando niveles bajos, todo esto en relación con la influencia del estrés ante un adecuado aprendizaje. Hay que considerar que en el ámbito educativo el estrés influye en los procesos de aprendizaje y memoria, porque cuando el estudiante está estresado la amígdala cerebral bloquea la absorción de estímulos sensoriales; es decir, que la información no será retenida, presentando dificultades con la memoria a largo plazo, lo que no permitirá que el alumno genere un adecuado aprendizaje, al no poder codificar y retener los conocimientos que va adquiriendo con el tiempo.

La tercera pregunta que se realizó fue: ¿Cuál es su nivel para manejar situaciones ansiosas al realizar tareas complejas?, en la siguiente Figura 4 se grafican los datos obtenidos. 
Figura 4.

Nivel para manejar situaciones ansiosas al realizar tareas complejas

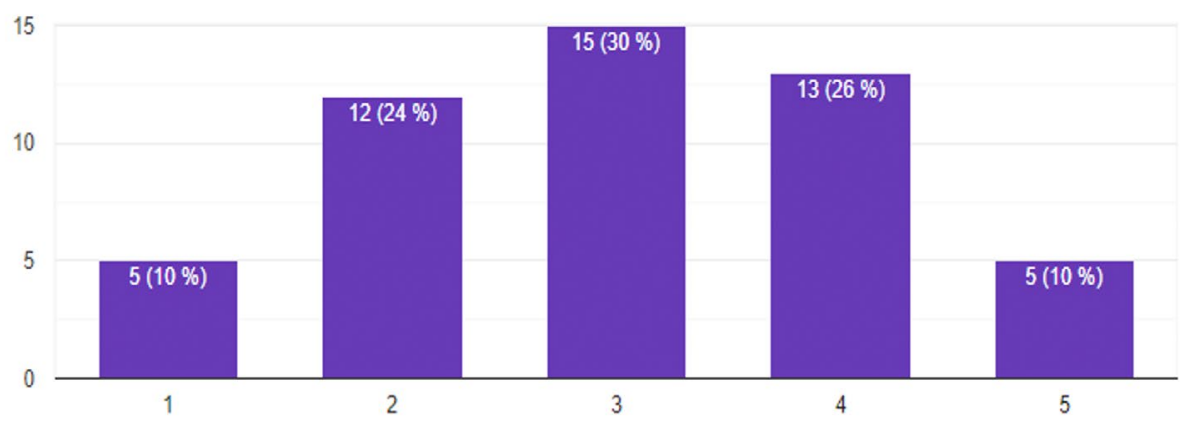

Se puede observar que el $30 \%$ respondió que su nivel de ansiedad sería 3 siendo neutral; un $26 \%$ con un nivel de 4 , el $10 \%$ corresponde a un nivel 5 , demostrando niveles altos; por otro lado, el $24 \%$ representa un nivel 2 y finalmente el $10 \%$ cuenta con un nivel 1 , indicando niveles bajos, todo esto en relación con el manejo de situaciones ansiosas al realizar tareas complejas. Una de las razones para que el estudiante no pueda manejar su ansiedad al realizar tareas es que estas tienen un grado alto de dificultad y también el no contar con el apoyo suficiente de los maestros se les dificulta poder comprenderla, además, al tener tiempos cortos en las entregas generan aún más ansiedad y no podrán manejar sus emociones adecuadamente o en otro casos algunos alumnos buscan el perfeccionismo y piensan que nada es lo suficientemente bueno lo que ocasionará problemas al presentar sus tareas y en su salud mental (Jadue, 2001).

En la Figura 5 se puede ver lo que respondieron los estudiantes a la cuarta pregunta: ¿Qué nivel de estrés le causa el utilizar herramientas tecnológicas para mejorar su aprendizaje?

Figura 5.

Nivel de estrés al utilizar herramientas tecnológicas para mejorar el aprendizaje

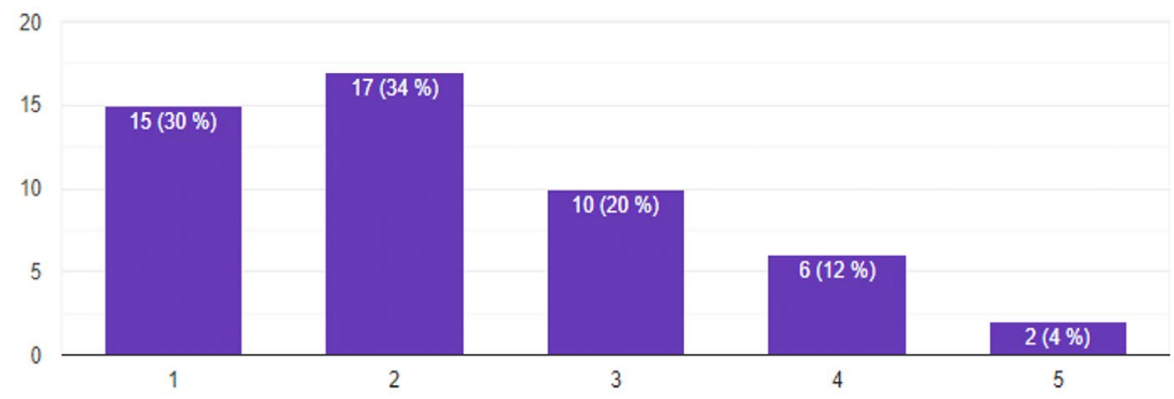

Se evidencia que el $34 \%$ respondió que su nivel de ansiedad sería 2, un 30\% con un nivel de 1 , siendo niveles bajos; el 20\% corresponde a un nivel 3, demostrando un nivel neutral; por otro lado, 
el $12 \%$ representa un nivel 4 y finalmente el $4 \%$ cuenta con un nivel 5 , indicando niveles altos, todo esto en relación con el estrés que causa el utilizar herramientas tecnológicas para mejorar el aprendizaje. En la actualidad se sabe que la tecnología brinda muchos beneficios en el ámbito educativo, al permitir un rápido aprendizaje, ya que en el Internet se pueden encontrar muchos artículos y libros que facilitan el adquirir conocimientos, a pesar de que la mayoría de personas se adaptan al uso de la tecnología a la perfección, hay personas que tienen inconvenientes al utilizar programas complejos, lo que les puede causar un estrés mental o en ciertos casos generar un estrés visual, afectándolos físicamente por el uso excesivo de aparatos tecnológicos.

Al implementar nuevas metodologías de estudio ¿Cuál es el nivel de ansiedad que le genera? Fue la pregunta cinco de la encuesta.

Figura 6.

Nivel de ansiedad al implementar nuevas metodologías de estudio

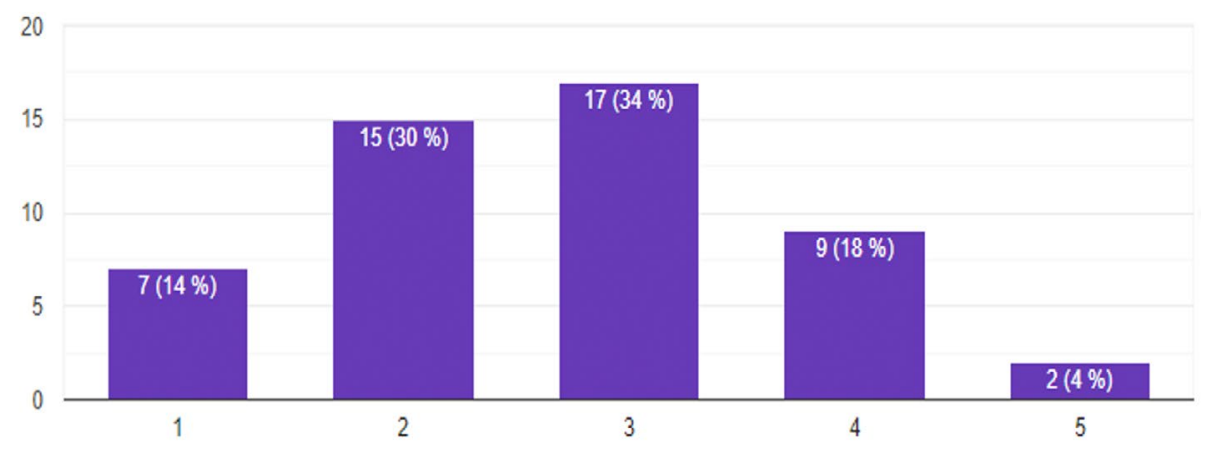

De acuerdo con la Figura 6 se observa que el 34\% respondió que su nivel de ansiedad sería 3, demostrando un nivel neutro; un $30 \%$ con un nivel de 2, el $14 \%$ corresponde a un nivel 1 , siendo niveles bajos; por otro lado, el 18\% representa un nivel 4 y finalmente el $4 \%$ cuenta con un nivel 5 , indicando niveles altos, todo esto en relación con la ansiedad al implementar nuevas metodologías de estudio. Con el pasar de los años se implementan metodologías en el proceso de aprendizaje con el fin de que el estudiante adquiera nuevas habilidades y conocimientos de manera eficiente y eficaz, aun cuando esto le favorece las nuevas metodologías tienen cierto nivel de complejidad, causando estrés al no poder aplicarlas, como por ejemplo, el modelo pedagógico conocido como aula invertida en la que el alumno debe estudiar en casa la materia para ponerla en práctica en las aulas de clase y así optimizar el tiempo.

La última pregunta que fue parte del cuestionario es la siguiente: si los docentes implementaran pausas activas durante las clases virtuales ¿Cuál sería su nivel de estrés para realizar las actividades en clase? 
Figura 7.

Nivel de estrés si los docentes implementan pausas activas en clases virtuales

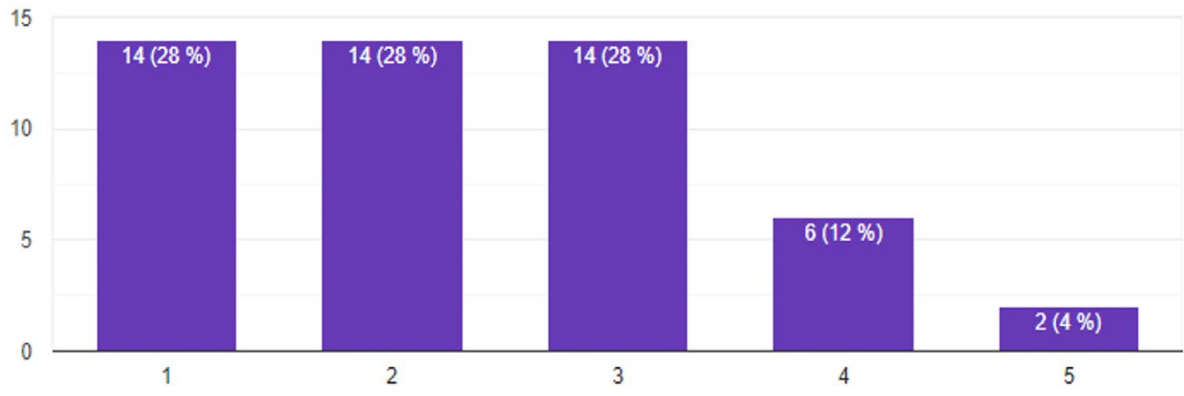

Como se puede ver en la Figura 7 , un $28 \%$ respondió que su nivel de estrés sería 3 , demostrando un nivel neutro; otro $28 \%$ muestra niveles bajos tanto en el 1 como en el 2; por otro lado, el $12 \%$ representa un nivel 4 y finalmente el $4 \%$ cuenta con un nivel 5 , indicando niveles altos, todo esto en relación si el estrés se reduciría al implementar pausas activas por los docentes en clases virtuales. Al implementar pausas activas, el estrés disminuye y en muchas ocasiones se lo puede prevenir al implementar descansos cortos para que los estudiantes recuperen energía física y mental, de manera que puedan tener un óptimo desempeño en sus actividades escolares, además de retener y entender los conocimientos adquiridos a lo largo de las clases.

\section{Conclusiones}

De acuerdo a los resultados se evidencia que la mayoría de estudiantes a los que se les aplicó la encuesta se mantienen en un nivel bajo y neutro de ansiedad ante las clases virtuales, generándoles posibles tensión por inconvenientes con la conectividad y el temor de posibles contagios por la pandemia actual; también se reveló que consideran que el estrés influye en el adecuado proceso de aprendizaje; además de esto, su manejo de ansiedad al realizar tareas complejas podría variar al ser estudiantes en otras modalidades de estudio; por otro lado, en relación al uso de herramientas tecnológicas y la implementación de nuevas metodologías, en su aprendizaje no son factores para generarles estrés; finalmente manifiestan que al tener pausas activas durante sus clases beneficiaria a la prevención y disminución del estrés.

Los problemas más comunes en la salud mental dentro de la educación son el estrés y la ansiedad, gran parte de los factores que influyen en estas patologías son las nuevas metodologías que se implementan en la educación, acorde a las situaciones que se presenten, tal es el caso de las clases virtuales por la pandemia de Covid-19, esta crisis dio un giro muy grande en la vida de las personas, ya que se tuvo que tomar medidas drásticas para evitar daños en la salud física y mental.

El impacto de la neurociencia ha permitido descubrir nuevos aportes como la neuroeducación y la neurolingüística en el ámbito educativo, influyendo en el proceso de aprendizaje. Partiendo 
de la psicología del aprendizaje al ser fundamental en la educación, busca generar nuevas estrategias que sean eficientes y eficaces para el desarrollo de las estudiantes y así puedan adquirir conocimientos de manera adecuada, teniendo en cuenta las metodologías actuales para que pueda estimular el desarrollo de su cerebro obteniendo nuevas habilidades para obtener un adecuado aprendizaje.

La importancia de la psicología del aprendizaje permite conocer teorías que ayudan a comprender de mejor manera el proceso de aprendizaje de los estudiantes, siendo la primera la teoría la conductista, que se basa especialmente en el condicionamiento y explica que las personas aprenden a través de la conducta que vendría a ser el estímulo; es decir, en el almacenamiento y la adquisición de información en donde el condicionar permite moldear el estímulo que se quiere aprender.

La siguiente es la teoría cognitivista, necesita de una maduración, y no solo cambios físicos, basándose en procesos mentales como el aprendizaje y el pensamiento empleando una metodología experimental; la tercera es la humanista, es un enfoque dentro de la psicología del "ser", más no del "tener", basándose en los valores de las personas, siendo libres y conscientes al tener una forma de afrontar los problemas de la vida con el fin de conseguir una óptimo desarrollo, estudiando al ser humano en totalidad; sin embargo, indica que todos son iguales y tienen el mismo nivel de aprendizaje y conocimiento; la última teoría es la del aprendizaje social, se establece de dos maneras a través de la conducta y el aprendizaje asociativo, centrándose en la observación para aprender de la experiencia y la imitación, esta postura es la de aprender por imitación por lo que al repetir un proceso observado podemos adquirir conocimientos, lo que permite retener información para ser usada en el desarrollo personal y social. 


\section{Referencias}

Aguilar, B., Cabrera, C., De Luna, C., Hernández, A., Hernández, A., y Juárez, X. (2020). Medicina en tiempos de Covid: estrés generado por educación virtual jóvenes de 18 a 25 años, de nivel profesional en una universidad privada del sur. Programa de Tutorías y Asesorías Académicas, 14.

Afkri, R., \& Hosseni, S. (2021). Investigating the Behavioral and Psychological effects of Virtual Education and staying at home during Corona Crisis on Elementary Students. Annals of the Romanian Society for Cell Biology, 25(4). https://www.annalsofrscb.ro/index.php/journal/article/view/5375

Araya, S., \& Espinoza, L. (2020). Aportes desde las neurociencias para la comprensión de los procesos de aprendizaje en los contextos educativos. Propósitos y Representaciones, 8(1). http://dx.doi.org/10.20511/ pyr2020.v8n1.312

Ardila, R. (2001). Psicología del aprendizaje. Siglo Veintiuno.

Blanco, L., Pérez, V., García, C., y Lobo, M. (2017). Neurociencia y Neuropsicología educativa. Ministerio de Educación.

Béjar, M. (2014). Neuroeducación. Padres y Maestros, (355), 49-53. https://doi.org/10.14422/pym.v0i355.2622

Bertrán, J. B. (2018). Cómo aprenden los seres humanos: una aproximación psicopedagógica. Ediciones UC. https://doi.org/10.2307/j.ctvkjb4jt

Bishop, J., \& Gagne, C. (2018). Anxiety, depression, and decision making: a computational perspective. Annual review of neuroscience, 41, 371-388. https://doi.org/10.1146/annurev-neuro-080317-062007

Cabeza, R., Albert, M., Belleville, S., Craik, F. I., Duarte, A., Grady, C. L., ... y Rajah, N. (2018). Maintenance, reserve and compensation: the cognitive neuroscience of healthy ageing. Nature Reviews Neuroscience, 19(11), 701-710. https://doi.org/10.1038/s41583-018-0068-2

Cáceres, Z., \& Munévar, O. (2017). Evolución de las teorías cognitivas y sus aportes a la educación. Actividad Física y Desarrollo Humano, 7, 2-9. https://doi.org/10.24054/16927427.v2.n2.2016.2408

Cano, S., Collazos, C., Flórez, L., Moreira, F., y Ramírez, M. (2020). Experiencia del aprendizaje de la Educación Superior ante los cambios a nivel mundial a causa del COVID-19. Campus Virtuales, 9(2), 51-59. http:// uajournals.com/ojs/index.php/campusvirtuales/article/view/734

Cepeda, F., Correa, M., Lozano, V., y Urquizo, Z. (2018). Análisis crítico del conductismo y constructivismo, como teorías de aprendizaje en educación. Open Journal Systems en Revista: REVISTA DE ENTRENAMIENTO, 4(1), 01-12. 
Escobar, M. (2015). Influencia de la interacción alumno-docente en el proceso enseñanza-aprendizaje. PAAKAT: Revista de Tecnología y Sociedad, (8) 2-7. http://www.udgvirtual.udg.mx/paakat/index.php/paakat/article/ view/230

Fajkowska, M., Domaradzka, E., y Wytykowska, A. (2017). Attentional processing of emotional material in types of anxiety and depression. Cognition and Emotion, 32, 1448-1463 https://doi.org/10.1080/02699931.201 $\underline{7.1295026}$

Gómez, M., \& Vázquez, E. (2018). Aportes de las Neurociencias a la Educación. TEPEXI Boletín Científico de la Escuela Superior Tepeji del Río, 5(10), 2-6. https://doi.org/10.29057/estr.v5i10.3310

Gómez, E., Navas, F., Aponte, G., y Betancourt, L. (2016). Metodología para la revisión bibliográfica y la gestión de información de temas científicos, a través de su estructuración y sistematización. Dyna, 81(184),158163. https://doi.org/10.15446/dyna.v81n184.37066

González, L. (2020). Estrés académico en estudiantes universitarios asociado a la pandemia por COVID-19. Espacio I+D, Innovación Más Desarrollo, 9(25), 159-168. https://doi.org/10.31644/IMASD.25.2020.a10

Granda, K. (2016). El condicionamiento operante como estrategia dentro del proceso de enseñanza en la actualidad educativa ecuatoriana. [Tesis de grado, Universidad Técnica de Machala]. Repositorio Digital de la UTMACH. http://repositorio.utmachala.edu.ec/handle/48000/9630

Hernández, R., Fernández, C., y Baptista, P. (2014). Metodología de la investigación. México. McGraw-Hill Interamericana.

Jadue, G. (2001). Algunos efectos de la ansiedad en el rendimiento escolar. Estudios pedagógicos (Valdivia), (27), 111-118. https://doi.org/10.4067/S0718-07052001000100008

López, C. (2018). La Educación Holística desde una Perspectiva Humanista. Revista Scientific, 3(8), 303-316. https://doi.org/10.29394/Scientific.issn.2542-2987.2018.3.8.16.301-318

Lovón, M., \& Cisneros, S. (2020). Repercusiones de las clases virtuales en los estudiantes universitarios en el contexto de la cuarentena por COVID-19: El caso de la PUCP. Propósitos y Representaciones, 8(SPE3), 588. https://doi.org/10.20511/pyr2020.v8nSPE3.588

Martín, S., \& Lafuente, V. (2017). Referencias bibliográficas: indicadores para su evaluación en trabajos científicos. Investigación bibliotecológica, 31(71), 151-180. https://doi.org/10.22201/iibi.0187358xp.2017.71.57814

Organización Mundial de la Salud. (2001). Fortaleciendo la promoción de la salud Mental. OMS, Hoja informática, No. 220. 
Pellón, R., Vázquez, M., Orgaz, C., Ortega, N., y Pérez, V. (2015). Psicología del aprendizaje. UNED - Universidad Nacional de Educación a Distancia.

Ramírez, J. (2020). Undergraduate Neuroscience Education: Meeting the Challenges of the 21 st Century. Neuroscience Letters, 739 https://doi.org/10.1016/j.neulet.2020.135418

Ramos, L. (s.f.). Nueva visión de la enseñanza con la Neuroeducación en el aula. Ceril. http://ceril.net/index. php/articulos?id=247

Rojas, D. (2017). Neurolinguística y aprendizaje. [Tesis de grado, Universidad Panamericana]. SCRIPTA https:// hdl.handle.net/20.500.12552/2926

Sandrone, S., \& Schneider, L. (2020). Active and distance learning in neuroscience education. Neuron, 106(6), 895-898. https://doi.org/10.1016/j.neuron.2020.06.001

Tan, Y., \& Yip, A. (2018). Hans Selye (1907-1982): Founder of the stress theory. Singapore Medical Journal, 59(4), 170. https://doi.org/10.11622/smedj.2018043

Terigi, F. (2016). Sobre aprendizaje escolar y neurociencias. Propuesta educativa, (46), 50-64. https://www.redalyc.org/pdf/4030/403049783006.pdf

Varona, F. (2016). Una mirada humanista a la educación estética de la sensibilidad humana. Aisthesis, (60), 111-128. https://doi.org/10.4067/S0718-71812016000200006

Vega, N., Flores, R., Flores, I., Hurtado, B., y Rodríguez, J. (2019). Teorías del Aprendizaje. XIKUA Boletín Científico de La Escuela Superior de Tlahuelilpan, 51-53. https://doi.org/10.29057/xikua.v7i14.4359

Yánez, P. (2016). El proceso de aprendizaje fases y elementos fundamentales. Revista San Gregorio, (11), 7279. https://dialnet.unirioja.es/servlet/articulo?codigo $=5585727$ 
Copyright (c) 2022 Lizbeth Aracely Toasa Guachi y Renato Mauricio Toasa Guachi

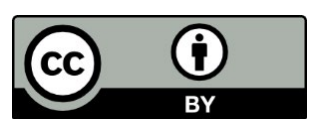

Este texto está protegido bajo una licencia internacional Creative Commons 4.0.

Usted es libre para Compartir-copiar y redistribuir el material en cualquier medio o formato - y Adaptar el documento - remezclar, transformar y crear a partir del material-para cualquier propósito, incluso para fines comerciales, siempre que cumpla las condiciones de Atribución. Usted debe dar crédito a la obra original de manera adecuada, proporcionar un enlace a la licencia, e indicar si se han realizado cambios. Puede hacerlo en cualquier forma razonable, pero no de forma tal que sugiera que tiene el apoyo del licenciante o lo recibe por el uso que hace de la obra.

\section{Resumen de licencia - Texto completo de la licencia}

